\title{
Le labyrinthe des ayants droit
}

Jean Jonassaint

\section{OpenEdition}

Journals

Édition électronique

URL : https://journals.openedition.org/coma/406

DOI : $10.4000 /$ coma.406

ISSN : 2275-1742

\section{Éditeur}

Institut des textes \& manuscrits modernes (ITEM)

\section{Référence électronique}

Jean Jonassaint, «Le labyrinthe des ayants droit », Continents manuscrits [En ligne], 3 | 2014, mis en ligne le 08 novembre 2014, consulté le 12 novembre 2021. URL : http://journals.openedition.org/ coma/406 ; DOI : https://doi.org/10.4000/coma.406

Ce document a été généré automatiquement le 12 novembre 2021.

\section{(c) (i) () $\Theta$}

Continents manuscrits - Génétique des textes littéraires - Afrique, Caraîbe, dispora est mis à disposition selon les termes de la licence Creative Commons Attribution - Pas d'Utilisation Commerciale - Pas de Modification 4.0 International. 


\title{
Le labyrinthe des ayants droit
}

\author{
Jean Jonassaint
}

1 Comment sortir du labyrinthe : Auteurs/ Légataires/ Héritiers/ Éditeurs/ Fiduciaires/ Dépositaires/ Photographes/ Traducteurs/ Interprètes/ Metteurs en scène/ Producteurs, ou même conservateurs et archivistes...?

Telle fut ma question initiale, tout à fait exploratoire, aucunement académique ou théorique, tentant de répondre à l'invitation de l'ITEM pour son colloque, «Sauver les manuscrits francophones: pour une charte de dépôt matériel et immatériel», à la fondation des Treilles ${ }^{1}$.

Me plaçant du point de vue du chercheur qui tente d'accéder à des documents dans des archives ou collections spéciales de bibliothèque à des fins de recherche, d'enseignement ou de publication scientifique, à partir d'exemples concrets, je voudrais plaider pour une simplification et une uniformisation des procédures d'accès et de diffusion. Tirant leçon d'une analyse des démarches de Grove Press pour le droit de traduire en anglais un extrait d'un poème de Saint-John Perse cité dans Une saison au Congo d'Aimé Césaire $(1966,1969)$; des problèmes de droits liés à la traduction anglaise d'un extrait de H'Éros-Chimères de Frankétienne $(2002,2009)$ ou à l'enregistrement sonore de la représentation de La Tragédie du Roi Christophe de Césaire $(1963,1964)$ à Dakar en 1966; de mes vaines tentatives d'obtenir copie d'un manuscrit anonyme des Mémoires de Boisrond-Tonnerre (1804) aux Archives Nationales d'Outre Mer (ANOM), parallèlement à cet appel pour plus d'ouverture des archives, je compte exposer les grandes lignes d'une possible politique pour un financement efficace des archives et bibliothèques spécialisées " publiques » ou " privées ».

Pourquoi ces guillemets à " privées » et "publiques", doivent se demander certains? Pour aller vite, simplement parce que des fonds publics ou parapublics, via la fiscalité et des subsides directs ou indirects, soutiennent toujours en partie les initiatives privées, tout comme le dévouement ou l'engagement personnels de certains fonctionnaires font la différence dans la performance des institutions dites publiques.

2 Ce détour fait, revenons à notre idée maîtresse d'analyser quelques situations complexes pour proposer une solution simple à la question des droits sur des documents archivés. 
Avant d'arriver aux propositions de solution, faisons donc quelques tours dans ce labyrinthe des ayants droit qui permettront de mieux saisir pourquoi simplification et uniformisation des procédures d'accès et de diffusion des documents conservés ou archivés devraient faciliter ou améliorer les conditions de travail de toutes les parties. En tout premier lieu, examinons deux traductions du français à l'anglais, chacune impliquant au moins deux niveaux de droits en plus de ceux des traducteurs: des extraits de Une saison au Congo d'Aimé Césaire $(1966,1973)$ et de H'Éros-Chimères de Frankétienne (2002).

\section{La traduction anglaise d'Une saison au Congo}

Ceux qui portent attention aux discours péritextuels seront frappés par une note en page de copyright de A Season in the Congo d'Aimé Césaire (1969), une traduction de Ralph Manheim publiée par Grove Press (qui, par ailleurs, est aussi l'éditeur américain de Samuel Beckett et de Jean Genet) : « The poem quoted on page 39 is by St-John Perse, Winds (Vents), copyright @ Gallimard 1960. This translation is printed by permission of Princeton University Press. »

Derrière ces deux lignes, se cache un processus en quatre étapes de demande de droit de publication que je souhaiterais, pour les besoins de la démonstration, retracer à grands traits à partir de documents d'archives :

- Demande d'information sur la citation de la part de Laura Furman, éditrice de Grove Press, au traducteur, Ralph Manheim (le 6 mars 1969);

- Réponse de Manheim détaillant les divers ayants droit : Gallimard, the Bolligen Series de la Bolligen Foundation, Princeton University Press (le 8 mars 1969);

- Lettre de demande de permission de Penelope Weiss, éditrice de Grove Press, à Princeton UP, accompagnée de la traduction de l'extrait faite par Manheim (le 2 septembre 1969) ;

- Lettre de Penelope Weiss à Manheim, l'informant que Princeton UP a donné le feu vert pour sa traduction, mais a suggéré certains changements qui sont notés sur les épreuves (le 14 novembre 1969).

Malheureusement nous n'avons pas ces galées dans les archives de Grove à Syracuse University, mais nous pouvons induire les corrections suggérées par l'analyse des différences entre le texte envoyé à Princeton UP, la copie pour la composition, et le texte publié. En effet,il apparaît deux différences mineures entre ces deux états. D'une part, une addition à la fin du quatrième vers, « with us ", qui se lit dans le texte publié : "[...] a greater light is born with us "; d'autre part, une substitution au cinquième vers de « is » par «keeps» doublée de l'addition d'un « us » qui nous fait passer de «the itching soul is still rebellious » au " the itching soul keeps us still rebellious ».

Cette démarche étalée sur huit mois (de mars à novembre 1969), grugeant de bonnes heures de travail, impliquant pas moins de quatre intervenants (Laura Furman et Penelope Weiss de Grove Press, Ralph Manheim, le traducteur, et Bill McGuire de Princeton UP), était-elle vraiment nécessaire? Fallait-il absolument l'aval des ayants droit américains? Manifestement non, d'autant plus que des années plus tard, Gayatri Chakravorty Spivak, dans sa traduction anglaise du même ouvrage publiée par Seagull Books (2010), ignore complètement les ayants droit de Saint-John Perse. Par contre, suivant en partie le texte français, elle met les mots du poète entre guillemets, alors que Manheim ne le fait pas, ni ne démarque typographiquement la citation par des italiques comme dans la première édition française de 1966 au Seuil ${ }^{2}$. On est même en 
droit de se demander pourquoi une nouvelle traduction anglaise si elle doit être soumise à l'imprimatur de l'éditeur de la première?

De plus, le même Manheim ne signale aucune autre citation d'Une saison au Congo, ni de La Tragédie du Roi Christophe. Pourtant dans cette dernière pièce, il y a plusieurs citations «silencieuses » de textes littéraires ou non. L'une des plus intéressantes a été mise en lumière par Rodney E. Harris dans L'Humanisme dans le théâtre d'Aimé Césaire (1973). Il s'agit d'une traduction presque mot à mot de cinq phrases de Black Majesty, the Life of Christophe, King of Haiti de John W. Vandercook que Césaire met dans la bouche de Christophe à la scène 7 du troisième acte, quand il s'adresse à Richard, comte de la Bande du Nord 3 .

Alors, pourquoi tant de prudence de la part de Grove pour l'extrait de Saint-John Perse? Je ne saurais vraiment répondre dans l'immédiat. Laissons ces questions ouvertes pour des échanges à venir... Du choc des idées jaillira sans doute quelque lumière.

\section{La traduction anglaise d'un extrait de $H^{\prime}$ Éros-Chimères}

7 Notre deuxième exemple, plutôt personnel, part du processus de traduction et de publication d'une version anglaise d'un extrait de H'Éros-Chimères de Frankétienne (2002), intitulé «MONOLOGUE INTÉRIEUR EN CHAMBRE IMAGINAIRE (Spirale dramatique en un acte) ", pour déterminer dans la chaîne des intervenants dans sa production qui seraient les ayants droit. Pour qui voudrait obtenir une autorisation de republication, ou mieux de réimpression de cette traduction (à la fois texte et image), une première étape s'impose : bien interpréter la note introductive qui la précède, notamment la première et la dernière phrase: "Excerpt from H'Éros-Chimères by Frankétienne (2002), p. 245-254. [...] Translation from French by Rachel Douglas with the collaboration of Jean Jonassaint, edited by Jean Jonassaint with the collaboration of Frankétienne $»^{4}$.

D'abord, il faut noter la constellation de collaborateurs (auteur / traducteur / éditeur) d'un procès de production en quatre étapes : Écrire / Traduire / Collaborer / Éditer...

Une des leçons à retenir du travail de Frankétienne et du processus de production de cette version anglaise d'un extrait de H'Éros-Chimères, c'est qu'un livre, ou tout imprimé en général, n'est pas un texte ${ }^{5}$. Le choix des caractères, la mise en page, le papier, le format, la reliure, et même l'éditeur ou la collection font de chaque imprimé un objet particulier. Ainsi, du tapuscrit de la traduction anglaise initiale de Rachel Douglas composé uniformément en Times New Roman 14 (état 1$)^{6}$ à la version anglaise de l'extrait de H'Éros-Chimères publiée, intégrant les passages calligraphiés ou typographiés avec des caractères de types et de tailles variés et les reproductions de tableaux (état 4), nous aboutissons à un autre ensemble topo / typographique qui donne, parfois, des valeurs différentes au texte ou à des fragments. Par exemple, lire la phrase « Where to start » dans une séquence de phrases donnée normalement de gauche à droite, puis de haut en bas, est fort différent de la lire dans une séquence qui encadre une image, rappelant un fotus (états 3 et 4). De même la lire dans l'état 4, formant un vrai rectangle de mots et d'images avec les deux autres phrases, implique une autre perspective de lecture, et induit même d'autres sens. Ce qui est démontré ici avec un livre poussant la typographie à ses limites peut être vérifié ou validé sur des ouvrages moins travaillés topo/typographiquement. Pour s'en convaincre, on n'a qu'à 
comparer, par exemple, l'édition originale de Mobile de Michel Butor (1962) avec sa reproduction dans la collection "L'Imaginaire " (1992), Oiseaux de Saint-John Perse dans son grand format de 1963 et sa réédition dans la collection «Pléiade » (1972). D'une édition à l'autre, l'expérience de lecture ou de perception de l'œuvre est différente. Dans le premier cas, on passe d'un texte qui se déploie majestueusement sur l'horizontal comme le continent américain d'Est en Ouest, à un texte écrasé ou corseté dans un rectangle vertical qui annule toute possibilité de lecture de la double page ; dans le second, du gras italique de taille 12 , ou plus, qui évoque un déploiement d'oiseaux dans un ciel, on aboutit au corps 10, à peine lisible, sur une page au format de poche qui force l'œil jusqu'à annuler en bonne part le plaisir du texte.

Qui plus est, les droits sur le texte diffèrent parfois des droits sur le livre ou l'imprimé. Les ouvrages illustrés, les éditions savantes et bilingues en sont des exemples les plus courants. Si les droits sur le texte au sens strict appartiennent à Frankétienne, comme ceux de sa traduction reviennent d'abord à Rachel Douglas, il n'est pas certain que ceux de la page imprimée intégrant texte / calligraphie / image appartiennent à Douglas ou Frankétienne. Le processus de mise en forme, mieux de mise en page (pour parler le langage typographique), ou de fabrication (pour parler la langue de l'imprimerie) est d'abord l'œuvre de Jonassaint. Par contre, les photos retravaillées des peintures reproduites sont d'une autre instance, le photographe, fort probablement Marie Andrée Étienne, la femme de l'auteur. Instance qui a été gommée dans la publication originale, comme la traduction gomme celle de l'(info)graphiste qui a aidé à la fabrication de l'objet-livre. Or, comme on peut le constater, sans cet effort collectif ou collaboratif, on n'aboutit pas au même résultat, comme le donnent à voir quelques états ou étapes intermédiaires de ce travail de transformation du français à l'anglais, du texte au texteimage, présentés en annexe. Par ailleurs, cet extrait de l'œuvre a été publié par une revue avec un numéro ISSN qui, de facto, a certains droits.

À qui donc s'adresser pour obtenir l'autorisation de reproduction de ces pages qui sont tant de l'ordre de l'image que du texte, en ce sens, tout à la fois image-texte et texteimage? Dans le cas de la reproduction photographique d'une œuvre d'art plastique, c'est fort connu et accepté, il y a généralement trois instances de droit: droits de l'artiste, du photographe, et des propriétaires ou dépositaires de l'œuvre (collectionneur, galerie d'art, musée...). Avec le copy-art, les enjeux peuvent se compliquer encore plus. Dans le travail de Frankétienne, il y a bien une part de copy-art que nous n'abordons pas ici, car n'impliquant pas des œuvres autres que celles de l'auteur-artiste, il n'introduit pas d'autres ayants droit. Par contre, dans l'éventualité que je fasse don des divers documents liés à la fabrication de ces pages à une bibliothèque spécialisée, selon les législations et pratiques actuelles, la question se pose de savoir qui peut autoriser leur reproduction en partie ou en totalité ou leur utilisation à des fins de recherche ou d'enseignement. En les publiant aujourd'hui, sans l'accord (formel) des diverses instances de production, viole-t-on les droits des auteur, traducteur, photographe, éditeurs (au pluriel, puisqu'il en a deux, par défaut de termes plus précis, appelons-les : l'éditeur scientifique ou directeur de publication et l'éditeur producteur ou marchand)? 


\section{Radiodiffusion d'une représentation de La Tragédie du Roi Christophe $(1963,1970)$}

11 Si pour la traduction anglaise de l'extrait de Frankétienne, nous faisons face à quatre entités civiles et institutionnelles - Frankétienne, l'auteur; Rachel Douglas, le traducteur ; Jean Jonassaint, le directeur de publication ; et le Journal of Haitian Studies, l'éditeur producteur -, dans le prochain cas analysé, la bande de la radiodiffusion de la représentation de La Tragédie du Roi Christophe (1961-1963, 1970) au Premier Festival Mondial des Arts Nègres à Dakar (1966), les intervenants et possibles ayants droit se multiplient. D'une part, contrairement aux exemples précédents, qui relevaient plutôt du droit d'auteur strict, dans ce cas il s'agit de droits d'auteur liés à des documents archivés. D'autre part, toute représentation publique d'une pièce implique minimalement, en plus des auteur et éditeur, un metteur en scène, une troupe de comédiens, et un producteur auxquels viennent s'adjoindre pour sa radiodiffusion: réalisateur, preneur de son, radiodiffuseur, pour ne rappeler que ceux-là.

12 Cette production de France Culture en collaboration avec la Radiodiffusion nationale du Sénégal, dans la foulée de la représentation par la défunte compagnie du Toucan à Dakar en 1966 de La Tragédie du Roi Christophe, est le "gala de France » au festival, comme noté clairement en page-titre de la brochure de présentation du spectacle ${ }^{7}$, donc jusqu'à un certain point propriété de l'État français. Mais le droit de propriété ici n'implique pas tous les droits, notamment celui de la propriété intellectuelle.

13 En fait, il s'agit d'une "version scénique " différente des "versions imprimées " connues dont le statut auctorial reste ambigu. Autrement dit, auteur et droits d'auteur ne sont pas aussi évidents qu'on pourrait le penser. Si le texte de base est de Césaire, le montage ou collage vu ou entendu est probablement du metteur en scène Jean-Marie Serreau comme le donne à penser un passage d'une lettre du 31 janvier 1970 de Ralph Manheim à Penelope Weiss sur le texte français qu'il a traduit. En effet, écrit-il:

Césaire expressly asked me to translate not the published book but the stage version used by Jean-Marie Serreau. But they were short of copies - it has never been published - and took mind away. I am pretty sure I can get you a copy now. I hope it has been retyped since because the thing I had to work with was a bloody mess.

Au mieux de nos connaissances, cette version scénique n'a pas été redactylographiée. Selon le témoignage de feue Jacqueline Lemoine (1923-2011), la troupe n'a jamais eu un tapuscrit intégral de la pièce remaniée par Césaire et Serreau, mais travaillait avec le texte imprimé de l'édition de 1963 et des bécquets que Césaire apportait pour les modifications ou ajouts (entrevue téléphonique du 22 septembre 2010 ${ }^{8}$ ). D'ailleurs, nous n'avons retracé que ces bécquets, et aucun tapuscrit de 1964-1966, à part celui de la télévision allemande qui est un abrégé du texte de 1963 fait par le directeur de Europa Studio ${ }^{9}$, M. Ottokar Runze, selon ce dernier, dans une entrevue téléphonique du 22 octobre 2010. 


\section{Transcription manuscrite anonyme des Mémoires pour servir à l'histoire d'Haïti de Boisrond-Tonnerre (1804)} directement vers la bureaucratie française, et plus largement vers ce qu'on pourrait appeler l'arbitraire des archivistes, conservateurs, bibliothécaires et autres dépositaires de patrimoine.

Avant d'aller plus loin dans notre exposé de ce cas, signalons que le terme " arbitraire " ne couvre pas qu'un aspect négatif. Au contraire, le pouvoir discrétionnaire de ces dépositaires peut jouer en faveur du chercheur. Mes propres expériences dans certaines bibliothèques françaises et américaines sont là pour prouver que ce pouvoir discrétionnaire est utilisé généralement avec bon sens, au profit de nos recherches.

D'ailleurs mes déboires dans mes démarches pour l'obtention d'une copie d'un manuscrit aux Archives Nationales d'Outre Mer (ANOM) ne reflètent pas tout à fait mon expérience avec cette institution comme exposé plus loin. Pour obtenir copie d'une transcription manuscrite anonyme des Mémoires pour servir à l'histoire d'Haïti de Boisrond-Tonnerre (1804) des ANOM, comme pour tout autre document, il y a une démarche à suivre. Il faut notamment compléter une fiche de «demande de réutilisation des données publiques", et soumettre certains documents et informations, entre autres: nom, adresse, et affiliation professionnelle pour les personnes physiques, en plus d'une reproduction d'une pièce d'identité ou du passeport; nom, adresse, capital social, numéro d'immatriculation, extrait du K-bis pour les sociétés ou les associations. De plus, le demandeur doit signer un engagement daté de n'utiliser ces documents qu'aux fins décrites dans la requête. C'est seulement après avoir fourni ces informations dont certaines fort personnelles qui, tombées entre de mauvaises mains, peuvent causer quelques nuisances irréparables, que les Archives étudieront votre demande en fonction de l'usage que vous comptez faire des données, donc approuveront ou non votre requête, puis vous feront une facture pour un usage limité à cinq ans. Déjà se pointent quelques problèmes. En cas de réimpression ou de réédition d'un ouvrage avec des données obtenues des ANOM six ans plus tard, doit-on renégocier le droit de reproduction? Si oui, à quel coût? Mais laissons en plan ces questions prospectives pour revenir à ma «demande d'utilisation des données publiques », pour reprendre le jargon des ANOM.

Suite à une prise de contact fort prometteuse avec l'archiviste de la section Caraïbe, M. Jacques Dion (qui m'a généreusement transmis en moins d'une journée les données pertinentes sur le document recherché) et la directrice des ANOM, Mme Cornede, qui m'a envoyé une lettre très cordiale le 6 juin 2013 semblant même me donner son accord, puisqu'accompagnée d'une photocopie de deux pages du manuscrit, j'ai rempli toutes les exigences des archives au 18 juin 2013.

Pourtant deux mois plus tard, je n'avais reçu aucune réponse à ma requête, malgré mes rappels par téléphone et email dont le dernier en date directement à $\mathrm{M}$. Jacques Dion remontait au dimanche 28 juillet 2013. Pour mémoire, citons ce mail :

Bonjour M. Dion : Le 18 juin dernier, j'ai transmis par email à Mme Andreoli, avec copies à vous-même, les documents demandés pour la demande de réutilisation des données publiques. J'ignore si vous avez eu mon email ou non, mais jusqu'à maintenant, je n'ai eu aucune réponse des Archives. Si possible, j'apprécierais grandement de savoir où en est ma demande. Cordialement, Jean Jonassaint 
Bien sûr, on peut penser que c'était l'été et, qu'en période de vacances, le personnel est réduit : il est assez normal que les délais soient plus longs. Mais, pourquoi ce silence? Difficile d'expliquer, qu'un an plus tard (en juillet 2014, révisant ma communication pour publication), nous soyons encore à la case départ, toujours en attente d'une réponse.

Quelle est la logique derrière ces exigences et retards bureaucratiques?

S'il y en a une, elle n'est pas des plus transparentes. Elle complique indûment le travail des chercheurs tout en coûtant cher aux institutions en temps-personnel à analyser des dossiers qui ne rapportent au bout d'un refus aucun revenu.

Comment sortir d'une telle quadrature?

\section{Propositions pour un meilleur accès au fonds d'archives publiques}

Pour aller vite, je propose d'établir :

- Un droit de consultation sans restriction contre des frais fixés à partir d'une grille universelle établie en fonction du type de chercheurs ou de la finalité des recherches (étudiants, professionnels, retraités, recherche pour publication commerciale ou scientifique, etc.);

- Un droit de reproduction sans restriction contre des frais fixés à partir d'une grille universelle établie en fonction du type de chercheurs (étudiants, professionnels, retraités), des types de documents (manuscrit, tapuscrit, imprimé ; document sonore ou audio-visuel, photographies, œuvres d'art, etc.), et du temps nécessaire au dépositaire pour leur reproduction. Par contre, les chercheurs pourraient perdre le droit de reproduire euxmêmes des documents; toute duplication devant être faite selon des normes rigoureuses facilitant en même temps sa digitalisation ou numérisation. Le rationnel ici est de réduire les coûts de mise en valeur supportés en partie par les usagers.

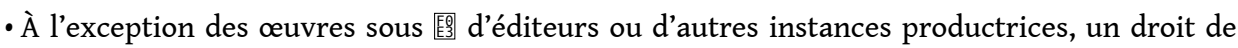
publication en tout ou en partie à des fins de recherche ou d'enseignement contre versement de redevances («royalties») aux ayants droit, s'il y en a, selon un tarif ou un pourcentage préétabli et uniforme pour tous. Sauf cas exceptionnel (possible libelle, par exemple), l'autorisation serait automatique. En cas d'incertitude sur les ayants droit ou de conflits entre divers ayants droit, en attente de règlement des litiges, les redevances seraient placées en fidéicommis.

- Les dépositaires ne devraient plus refuser, bloquer ou retarder l'accès à des documents sous des prétextes fallacieux. Par contre, à leur discrétion, mais selon certains paramètres minimaux à définir, ils pourraient exempter les chercheurs au Sud comme ceux n'ayant pas les ressources financières pour acquitter ces frais.

- Cet ensemble de propositions ayant pour but de sortir les documents archivés de la logique du marché et de l'arbitraire des ayants droit ou dépositaires, mais aussi de sortir les archives de la bureaucratie dévoratrice des ressources et improductive pour tous, il doit impliquer forcément une rupture avec les politiques actuelles d'acquisition contre débours. Les institutions vouées à la conservation des patrimoines culturels, notamment écrits ou imprimés, n'achèteraient plus des fonds, coupant ainsi court à toute une chaîne spéculative, et à une concentration des patrimoines littéraires dans les grandes institutions du Nord ${ }^{10}$.

- Un accord entre toutes les parties, qu'elles soient privées ou publiques, pour en finir avec la marchandisation des patrimoines culturels mondiaux, stipulant que les propriétaires ou 
ayants droit de biens patrimoniaux souhaitant que leurs fonds soient conservés et mis en valeur par des archives, des bibliothèques spécialisées ou même des musées, devraient les céder sans restriction aucune sur la consultation ou la diffusion (hormis les œuvres sous contrat), et sans compensation autre que des exemptions d'impôts ou un dédommagement forfaitaire pour ceux ne pouvant bénéficier de tels arrangements fiscaux. Ces éventuels dédommagements seraient fonction de paramètres préétablis, non négociés au cas par cas. Par ailleurs, les ayants droit pourront, comme il se pratique déjà au Québec, échanger leurs exemptions d'impôts contre paiement avec des tiers, mais ne recevront aucune autre compensation financière des institutions qu'une part des "royalties» et des droits de consultation ou de reproduction que leur fonds génèrent. Par contre, s'ils veulent imposer leurs propres règles sur l'utilisation de leurs fonds, ils devront défrayer eux-mêmes les coûts de traitement et de conservation. L'État n'aura pas à payer partiellement ou entièrement pour conserver des biens patrimoniaux au profit de quelques individus - les ayants droit qui empocheraient des dividendes, alors que ceux qui paient en partie, sinon entièrement la note, les contribuables, sont privés de l'usage de ces biens.

- L'économie faite sur les acquisitions libérerait des fonds pour conserver davantage de documents et soutenir leur diffusion sur les plans scientifique et éducatif, mais aussi pour financer la campagne de sensibilisation et d'éducation, tant sur le plan national qu'international, sur l'importance de la sauvegarde, de la conservation et de la valorisation des biens culturels patrimoniaux, notamment les plus fragiles comme les manuscrits, que requiert cette nouvelle politique pour bien s'implanter. Elle permettrait également de libérer des ressources pour mieux évaluer les offres de dons. Car rompre avec la marchandisation des patrimoines culturels n'implique pas un refus d'évaluer leurs intérêts intellectuels, artistiques, scientifiques ou autres. Au contraire! De plus, on ne peut pas tout conserver, et encore moins mettre tout en valeur, il faudra encore toujours choisir, sans dogmatisme certes, mais avec rigueur, selon des critères bien établis qui peuvent et doivent évoluer pour répondre aux besoins des chercheurs et plus largement du corps social.

- Enfin, une partie des revenus générés par les frais ou redevances perçus sur la consultation, la reproduction ou la publication des documents archivés devrait être placée dans un fonds commun géré par un organisme paragouvernemental ou associatif en vue d'aider les institutions moins nanties, notamment au Sud.

\section{En guise de conclusion}

L'idée de départ était d'exposer quelques situations complexes pour proposer des pistes de solution simples à la question des droits sur des documents sauvegardés par des institutions publiques ou privées. Y suis-je arrivé ? L'avenir le dira. Mais un premier constat s'impose. Par exemple, l'appel du collectif de Treilles dont je suis l'un des signataires, "Attention, manuscrits en péril!», ne tient aucunement compte d'un de mes arguments centraux, à savoir sortir de l'arbitraire des ayants droit. En effet, il stipule vouloir « Conserver matériellement et numériser les manuscrits littéraires dont la valeur est reconnue, traiter et restaurer ceux qui seraient endommagés selon un protocole technique approprié, leur offrir des conditions de protection pérenne par un dépôt dans des lieux présentant toutes les garanties de sécurité requises et dans un cadre juridique préservant les droits de chaque déposant à garder l'entière possession des documents sauvegardés. » C'est nous qui soulignons ce droit entier de propriété privée sur un bien sauvegardé avec des ressources publiques qui va à l'encontre de toute logique, et la tendance actuelle des institutions de conservation à refuser des dépôts 
qui, à moyen ou long terme, peuvent être retirés avec toutes les pertes que de tels retraits impliquent. Sur ce point, j'ai de fortes réserves, tout en adhérant aux principes généraux de ce document, et même à l'idée d'accorder un certain droit de regard aux ayants droit sur leurs dons patrimoniaux. Mais, en aucun cas, il ne peut s'agir de leur laisser « l'entière possession des documents sauvegardés». Ce serait contraire à toute bonne gestion des fonds publics, et aller à l'encontre de notre temps où l'État, de plus en plus, demande que les biens produits avec ses fonds soient, dans la mesure du possible, le plus largement accessibles à tous. C'est le sens même d'un «Memorandum " du 22 février $2013 \mathrm{du}$ bureau des politiques scientifiques et technologiques de la Maison Blanche (Office of Science and Technology Policy), «Increasing Access to the Results of Federally Funded Scientific Research ", aux responsables des ministères et agences fédéraux ${ }^{11}$. C'est aussi, sans contredit, le sens de la politique de démocratisation culturelle prônée en France avec la fondation même du ministère des Affaires culturelles dont le décret de constitution en 1959 stipulait qu'il a pour «mission de rendre accessible les œuvres capitales de l'humanité et d'abord de la France, au plus grand nombre possible de Français $»^{12}$.

Par contre, sur le plan de la diffusion, l'appel rejoint mes propositions, quand il préconise de "Valoriser ces manuscrits littéraires et dans une logique de bien public mondial, les rendre accessibles à tous, sous la forme la mieux adaptée à leur lecture et la plus favorable à leur diffusion, dans le respect de la propriété morale et intellectuelle, ainsi que du droit des personnes, des propriétaires et de la communauté des lecteurs. » C'est nous qui soulignons encore pour soulever une question: les rédacteurs ont-ils conscience des contradictions internes à ce dernier point? Dans cette affaire où, plus particulièrement, l'intérêt public et celui des ayants droit ("vendeurs ", « donateurs " ou " dépositeurs » toujours " propriétaires ») ne convergent pas toujours, on ne peut pas plaire à tous, il faut trancher. Certes, c'est là que le bât blesse. Mais ménager la chèvre et le chou ne peut conduire qu'à une impasse tant pour la préservation et la conservation des manuscrits et autres biens patrimoniaux que pour leur diffusion, sinon leur consultation à des fins de recherche et d'enseignement. Simplifier les règles, couper court aux cas d'espèces, avoir une politique universel d'accès, du moins dans ses grands principes - car il faut faire avec les législations et ressources ou contextes nationaux -, priorisant nettement la recherche et l'enseignement, ce sont les seules voies d'avenir, les seules justifiant les sommes colossales qu'impliquent la préservation et la conservation patrimoniales.

\section{NOTES}

1. Ce texte est une version revue et augmentée d'une communication au «Colloque des Treilles » sur les manuscrits en péril organisé par l'ITEM, du 2 au 6 septembre 2013. Il se fonde sur certaines idées, nées d'une praxis de recherche et d'enseignement, plutôt que sur des travaux académiques au sens strict : texte d'alerte donc à discuter, à commenter, à corriger en vue de possibles actions à prendre. Cette version a grandement bénéficié des échanges intenses et riches du Colloque de Treilles dont je tiens à remercier les organisateurs et hôtes, particulièrement 
Mmes Anne Bourjade, Valérie Dubec-Monoyez, Claire Riffard et M. Pierre-Marc de Biasi. Elle tire aussi profit d'une expérience de négociations pour des droits d'utilisation d'œuvres musicales pour une production audio-visuelle réalisée en mai 2014 dans le cadre de mon séjour à l'Institut d'Études Avancées de Nantes que je remercie pour son soutien.

2. Voir respectivement : Césaire, A Season in the Congo (New York: Grove Press, 1969), p. 39 et A Season in the Congo (Calcutta et Londres : Seagull Books, 2010), p. 53-54.

3. Voir entre autres: Vandercook, Black Majesty, the Life of Christophe, King of Haiti (New York: Harper, 1928), 187-189 ; Harris, L'Humanisme dans le théâtre d'Aimé Césaire (Sherbrooke : Naaman, 1973), p. 117-118.

4. Voir Frankétienne : H'Éros-Chimères, Journal of Haitian Studies 14.1 (2008), p. 159.

5. Sur cette question, voir en autres l'article de Jacques Anis, « Le livre : territoire et frontières ", in Louis Hay et al., De la lettre au livre : sémiotique des manuscrits littéraires (Paris : édition du CNRS, 1989), p. 169-193.

6. Voir en annexe, des extraits des quatre états discutés qui sont autant d'étapes de production :

1) la traduction révisée de Rachel Douglas; 2) la traduction de Rachel Douglas révisée et commentée par JJ ; 3) un premier montage du texte, des images et des calligraphies de Frankétienne par JJ ; 4) le prêt à photographier réalisé par l'infographiste du Journal of Haitian Studies suivant les directives de JJ.

7. Dans une entrevue parue le 9 décembre 2010 dans Le Quotidien de Dakar, Jacqueline Scott, qui interpréta le rôle de Madame Christophe, tant à Salzburg (1964) qu'à Dakar (1966), rappelle que la représentation de La Tragédie du Roi Christophe au Premier Festival Mondial des Arts Nègres était « le spectacle [...] offert par la République française ». Voir : <http://xalimasn.com/deces-de-laveuve-de-lucien-lemoine-les-ultimes-confessions-de-jacqueline/>, « Décès de la veuve de Lucien Lemoine : les ultimes confessions de Jacqueline ".

8. Sauf indication contraire, les entrevues mentionnées dans cet article ont été réalisées par JJ.

9. Filiale du puissant groupe allemand Bertelsmann, Europa Studio fut le producteur de Serreau pour les représentations de Salzbourg, Venise et ailleurs en Europe en 1964, et co-producteur de la version filmique de La Tragédie du Roi Christophe, diffusée par la télévision allemande le 20 janvier 1965.

10. Certains archivistes et bibliothécaires consultés pensent qu'une telle proposition n'est pas tout à fait réaliste. Sans vouloir débattre de cette question fort hypothétique, seul l'avenir permettra vraiment de trancher, il me semble qu'une telle position de principe réduira considérablement la demande, par conséquent, en bonne logique de marché, fera chuter les prix. Bien sûr, cette politique n'entraînera pas la disparition de tous les amateurs et spéculateurs sur ce marché, mais en réduira considérablement le nombre. Par ailleurs, si les grandes institutions privées adhèrent à ce principe, il est évident que la pression du marché chutera encore plus. Une telle éventualité n'est pas utopique. On n'a qu'à se rappeler la fronde des grandes bibliothèques universitaires américaines contre les géants des revues scientifiques - qui ont dû faire des concessions sur leur politique d'accès et les prix - dont le «Faculty Advisory Council Memorandum on Journal Pricing" de Harvard University du 17 avril 2012 <http:// isites.harvard.edu/icb/icb.do?keyword=k77982\&tabgroupid=icb.tabgroup143448> donne bien la mesure. De plus, une politique aussi claire et radicale découragera le marchandage de certains ayants droit, sachant que la surenchère sur ces biens est fort limitée. En bref, je pense que c'est sans doute la meilleure voie pour l'avenir.

11. Voir : John P. Holdren, Memorandum for the Heads of Executive Departments and Agencies, à <http://www.whitehouse.gov/sites/default/files/microsites/ostp/

ostp_public_access_memo_2013.pdf>.

12. Voir: Historique du Ministère de la Culture, disponible en ligne à < http:// www.culture.gouv.fr/culture/historique/rubriques/creationministere.htm>. 


\section{RÉSUMÉS}

À partir d'une analyse des difficultés rencontrées ou posées pour consulter, reproduire ou diffuser (totalement ou partiellement) des documents écrits ou audio-visuels d'Aimé Césaire, de Saint-John Perse, Frankétienne et Boisrond-Tonnerre, qui sont archivés ou non, cet article appelle à la simplification et l'harmonisation des règles d'accès aux archives, et la mise en place d'un ensemble de procédures pour traiter avec les titulaires de droit d'auteur qui soit efficace et équitable pour toutes les parties.

In the wake of an analysis of the difficulties encountered or envisioned to consult, reproduce, distribute or broadcast (fully or partially) written or audio-visual documents by Aimé Césaire, Saint-John Perse, Frankétienne and Boisrond-Tonnerre, which are archived or not, this article calls for a simplification and an harmonization of the rules to access to archives, and the establishment of a standard set of procedures to deal with copyright holders that be efficient and fair for all parties.

\section{INDEX}

Mots-clés : archives, manuscrit, édition, Saint-John Perse, Boisrond-Tonnerre, ayant droit, charte, Césaire, Frankétienne

Keywords : manuscript, Saint-John Perse, edition, right holder, Boisrond-Tonnerre, charter, Césaire, Frankétienne, archives

\section{AUTEUR}

JEAN JONASSAINT

Syracuse University 